\title{
Direct solid surface fluorescence spectroscopy of standard chemicals and humic acid in ternary system
}

\author{
S. Mounier ${ }^{\mathrm{a}, *}$, G. Nicolodelli ${ }^{\text {b }}$, R. Redon ${ }^{\text {a }}$, D.M.B.P. Milori ${ }^{\mathrm{b}}$ \\ a Laboratoire PROTEE, Université de Toulon, CS 60584-83041 Toulon Cedex 9, France \\ ${ }^{\mathrm{b}}$ EMBRAPA Instrumentação, Rua XV de Novembro, 1452 - Centro, São Carlos, SP, Brazil
}

\section{A R T I C L E I N F O}

\section{Article history:}

Received 21 July 2016

Received in revised form 21 December 2016

Accepted 7 January 2017

Available online 18 January 2017

\section{Keywords:}

Front face fluorescence spectroscopy

Solid state

Mixture

\begin{abstract}
A B S T R A C T
The front face fluorescence spectroscopy is often used to quantify chemicals in well-known matrices as it is a rapid and powerful technique, with no sample preparation. However it was not used to investigate extracted organic matter like humic substances. This work aims to fully investigate for the first time front face fluorescence spectroscopy response of a ternary system including boric acid, tryptophan and humic substances, and two binaries system containing quinine sulfate or humic substance in boric acid. Pure chemicals, boric acid, tryptophan, quinine sulfate and humic acid were mixed together in solid pellet at different contents from 0 to $100 \%$ in mass. The measurement of excitation emission matrix of fluorescence (3D fluorescence) and laser induced fluorescence were then done in the front face mode. Fluorescence matrices were decomposed using the CP/PARAFAC tools after scattering treatments. Results show that for 3D fluorescence there is no specific component for tryptophan and quinine sulfate, and that humic substances lead to a strong extinction effect for mixture containing quinine sulfate. Laser induced fluorescence gives a very good but non-specific related response for both quinine sulfate and tryptophan. No humic substances fluorescence response was found, but extinction effect is observed as for 3D fluorescence. This effect is stronger for quinine sulfate than for tryptophan. These responses were modeled using a simple absorbance versus emission model.
\end{abstract}

(C) 2017 Elsevier B.V. All rights reserved.

\section{Introduction}

The fluorescence spectroscopy is a useful, qualitative and quantitative technique used in lot of areas like agroalimentary, environment, agricultural, pharmaceutical, biological or medical domain. The most common use of the fluorescence spectroscopy concern molecules in solution, as the relation of the fluorescence response is directly related to the concentration of the fluorescent molecules. When individual spectrum is known, the technique shows a high quantitative and selective potential that is used for analytical purpose. Since a decade, the use of the total luminescence spectra or emission excitation matrix (EEM) allows the determination of several fluorescent compounds in a mixture using peak picking correlation [10]. This method is robust for mixture of pure molecules, diluted and with independent spectra behavior but often the problems of spectral overlapping and inner filter effect (IFE) raised in natural or uncontrolled samples. Hence, the EEM spectra need complex data treatment to separate independent sources and scattering processes [2,3]. It generally consists about the elimination of Raman and Rayleigh scattering (first and second order), the inner filter effect correction and the source separation. The scattering peaks are

\footnotetext{
* Corresponding author.

E-mail address: mounier@univ-tln.fr (S. Mounier).
}

generally suppressed by Zeep method [4], inner filter effect problems are solved by absorbance correction [13], moderate dilution (CDA) [17] to high dilution [21].

The interest for solid fluorescence spectroscopy in environmental purpose is strong because it does not exist rapid and robust method to fully characterize organic matter in solid sample of soil. Such measurement would allow, on one hand, field measurements for carbon stock evaluation, pollution quantification and, on another hand, on line quantification or verification like active compound concentration $[5,4]$ or food process control [2]. As natural soil is a too complex, this work aims to investigate the humic substance response in simpler medium.

Based on this, front face fluorescence spectroscopy (FFFS) could be a powerful method to analyze solid systems without any preparation or extraction. Fluorescence spectroscopy of solids by FFFS has been investigated highly in food preparation and pharmaceutical domain. Satisfactory results were obtained on milk [14], cheese [15], meal or medicals [3] and some extend to environmental samples have been done as on sediments [8], sewage [20], compost or synthetic soils [5,4]. Theses samples are complex mixture and no information are known about the molecular composition of the organic matter inside. Moreover, if some systematic investigations were done on controlled system, none was done on a full ternary system containing mineral matrix, standard of humic substances and model molecules. Moreover, to our knowledge 
no physical or empirical fluorescence model exists in the case of heterogeneous surface fluorescence, and only a Monte Carlo model was done for a light scattering medium [16].

The tryptophan (TRP) is an amino acid present in all the living macromolecules. Its spectroscopic properties in solution [22] and in solid state [20] are well known and used in a large extend [21]. The absorbance of tryptophan presents two peaks at 220 and $280 \mathrm{~nm}$. Its fluorescence quantum yields are higher than the phenylalanine and tyrosine ones [9]. Moreover, the TRP emission spectra has the property to shift in emission depending on the polarity of its environment in solution [22] and is located between 310 and $360 \mathrm{~nm}$ in protein environment [24]. In water, when the molecule is free, the maximum emission is located at $350 \mathrm{~nm}$ [22]. In solid matrix, the maximum emission of the TRP was found at $340 \mathrm{~nm}$ [20], with two excitation wavelength (250 and $280 \mathrm{~nm}$ ) like in solution, but this maximum can vary from 305 to 400 in biological environment [15]. Quinine Sulfate (QS) is a well-known fluorescent molecule that is used like a standard in fluorescence domain [18], particularly in environmental purpose where it is used to make the Quinine Sulfate Unit (QSU) for liquid samples, in the way to compare the different spectrofluorimeter results [11]. Standards of humic substances are generally used in solution as model of a part of the soils organic matter. In solution they fluoresce for an excitation of $250 \mathrm{~nm}$ and $350 \mathrm{~nm}$, with an emission from 400 to $450 \mathrm{~nm}$, depending on soils origin $[12,25]$. Few studies were done on their fluorescence in solid state [20] and no exhaustive exploration was done until now. This work aims to fully investigate for the first time FFFS response of a ternary system including boric acid, tryptophan and humic substances, and two binary systems containing quinine sulfate or humic substances in boric acid. Furthermore, it concerns the understanding of the interaction between standard fluorescent molecules and solid matrices like boric acid in presence of humic substances.

\section{Material and Method}

\subsection{Sample Preparation}

Samples were prepared with analytical powder grade: tryptophan (TRP, Merck), quinine sulfate (QS, Acros Organics), boric acid (BA, Fisher Chemical) and humic acid (HA, humic acid, sodium salt, technicalALDRICH) which was technical grade. TRP and BA are white solid, while QS is yellowish powder and HA is a dark-brown one. Amounts of each product were weighted to obtain the wanted content of each component in the range of 0 to $100 \%$ of the total weight, stepped by $10 \%$ (Fig. 1). The different amounts were grounded smoothly during 5 min until homogeneous powder was obtained. The resulting solid mixture ( $300 \mathrm{mg}$ ) was then pressed under 8 tons $\cdot \mathrm{cm}^{-2}$ to give a solid pellet. Each pellet was conserved in dry room, in the dark and in individual box. For the HA-TRP-BA ternary experiment 54 solid samples were done and ten measurements were done on each one, making on each pellet face five EEMs and rotating each time the pellet with an $2 \pi / 5$ angle to avoid surface effect. This data set is called (HA-TRP-BA\} in this work and composition mixture resumed in Fig. 1. For QS, two binary system were additionally prepared with amount of BA and amount $\mathrm{HA}$, and ten EEMs were obtained using the same protocol than HATRP-BA system. The resulting data set of EEM is called $\{\mathrm{QS}-\mathrm{BA}\} ;\{\mathrm{QS}-$ $\mathrm{HA}\}$ in this work. The $\{\mathrm{HA}-\mathrm{TRP}-\mathrm{BA}\}$ experiment will be labeled by the $\mathrm{T}$ index and the $\{\mathrm{QS}-\mathrm{BA}\} ;\{\mathrm{QS}-\mathrm{HA}\}$ experiments labeled by the $\mathrm{B}$ index.

\subsection{Excitation Emission Matrix Measurement}

\subsubsection{Laser Induced Fluorescence}

The laser induced fluorescence (LIF) was measured with a portable LIF system (lab-made equipment developed by EMBRAPA Instrumentation) equipped with a $405 \mathrm{~nm}$ laser source [23]. An optical fiber was coupled with a solid sample holder. Each face of each pellet was measured in the following conditions: emission wavelength range from

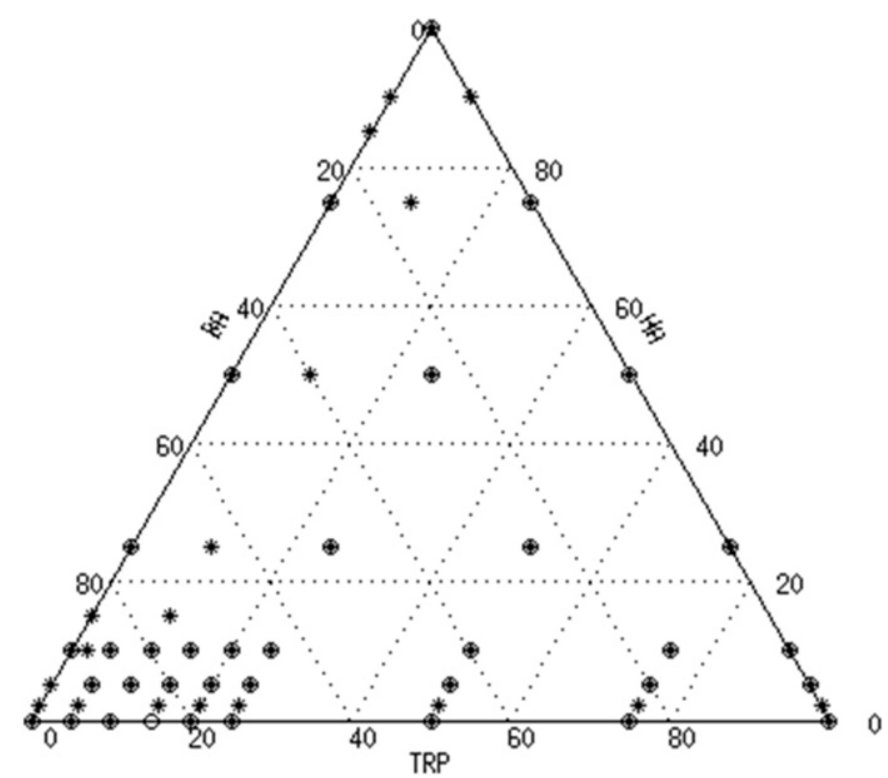

Fig. 1. Experiment plan of samples preparation for the \{TRP-BA-HA \} ternary system. Stars $\left({ }^{*}\right)$ represent ten measurements at different angles and faces, and circle (o) single two face measurement. Each side of the triangle represents the variation form 0 to $100 \%$ of the labeled molecule in the ternary system (down side the tryptophan (TRP), right side the boric acid (BA), and left side the humic acid (HA).

440 to $800 \mathrm{~nm}$, integration time $1 \mathrm{~s}$, boxcar 4 and average 5. For each measurement the integration of the area under the fluorescence emission curve from 440 to $800 \mathrm{~nm}$, (IF $\left.{ }_{405}\right)$, is calculated.

\subsection{Excitation Emission Matrix Acquisition}

The fluorescence EEM were measured on a HITACHI F4500 spectrofluorimeter using the solid holder module and measuring the pellet directly without the quartz glass. The excitation range was from 200 to $600 \mathrm{~nm}$ varying every $10 \mathrm{mn}$ and with an excitation slit of about $1 \mathrm{~nm}$. The corresponding emission spectra were acquired from 200 to $600 \mathrm{~nm}$ with a scan speed of $1200 \mathrm{~nm} \cdot \mathrm{min}^{-1}$ and a slit of $1 \mathrm{~nm}$. Extraction of the $10 \mathrm{~nm}$ stepped emission was obtained by the FL-Solution software. The photomultiplicator tension was fixed $950 \mathrm{~V}$ and the integration time set at $0.1 \mathrm{~s}$.

\subsection{CP/PARAFAC Analysis}

In the way to determine the contribution of the component to the EEM, the CP/PARAFAC algorithm was used [7,26]. First all the EEMs were cleaned from the diffusion signals: the Rayleigh by cutting the diffusion band ( $20 \mathrm{~nm}$ ) and the Raman from first and second order by applying the Zeep procedure [27]. The CP/PARAFAC algorithm allows the decomposition of a dataset of matrices based on a trilinear model, considering that all EEM is constituted by a linear combination of independent components and optimizing the residue between real EEM matrices and the components. One can note that the components are found without precognition of the spectra shape. The correct number of component needed to model the dataset is defined by evaluating the CORCONDIA score. This parameter falls down under 50\% when extra or usefulness components are present. Hence, users have to test a range of model, i.e. number of components, to detect the best number of component [6]. In this work, decomposition investigation was done from two to six components and the higher number of component giving a CORCONDIA test over $60 \%$ was selected as the optimal component number [19]. No outliers were present in the data. CP/PARAFAC was applied separately on the \{TRP-BA-HA data set system and on the joined $\{\mathrm{QS}-\mathrm{HA}\} ;\{\mathrm{QS}-\mathrm{BA}\}$ dataset systems. It was found three components both for the $\{$ TRP-BA-HA $\}$ system and for the $\{Q S-B A\} ;\{Q S-H A\}$ systems. 


\section{Results and Discussions}

\subsection{EEMs Results}

Depending of the mixture composition, several trends were observed in the EEM response (Supplementary material, (Fig. 6)). When TRP is predominant, the EEMs show two massive peaks after scattering correction: the first one is located at $\left(\lambda_{\text {ex }} / \lambda_{\text {em }}\right)=250 / 350 \mathrm{~nm}$ and the second one at $\left(\lambda_{\mathrm{ex}} / \lambda_{\mathrm{em}}\right)=250 / 600 \mathrm{~nm}$ (Supplementary material, (Fig. 6)). This second peak is related to the second order image of the first peak of TRP. When QS is predominant a fluorescence response occurs at $\left(\lambda_{\mathrm{ex}} / \lambda_{\mathrm{em}}\right)=300 / 400-450 \mathrm{~nm}$ showing a large emission range.

The decomposition by CP/PARAFAC was done for 2 to 6 components on \{TRP-BA-HA \}and gives the best result, i.e. smaller CORCONDIA over $60 \%$, for 3 components. These components are presented in Fig. 2 and will be named $\mathrm{C}_{\mathrm{T}}, \mathrm{C}_{\mathrm{T}}$ and $\mathrm{C} 3_{\mathrm{T}}$ respectively. The first component
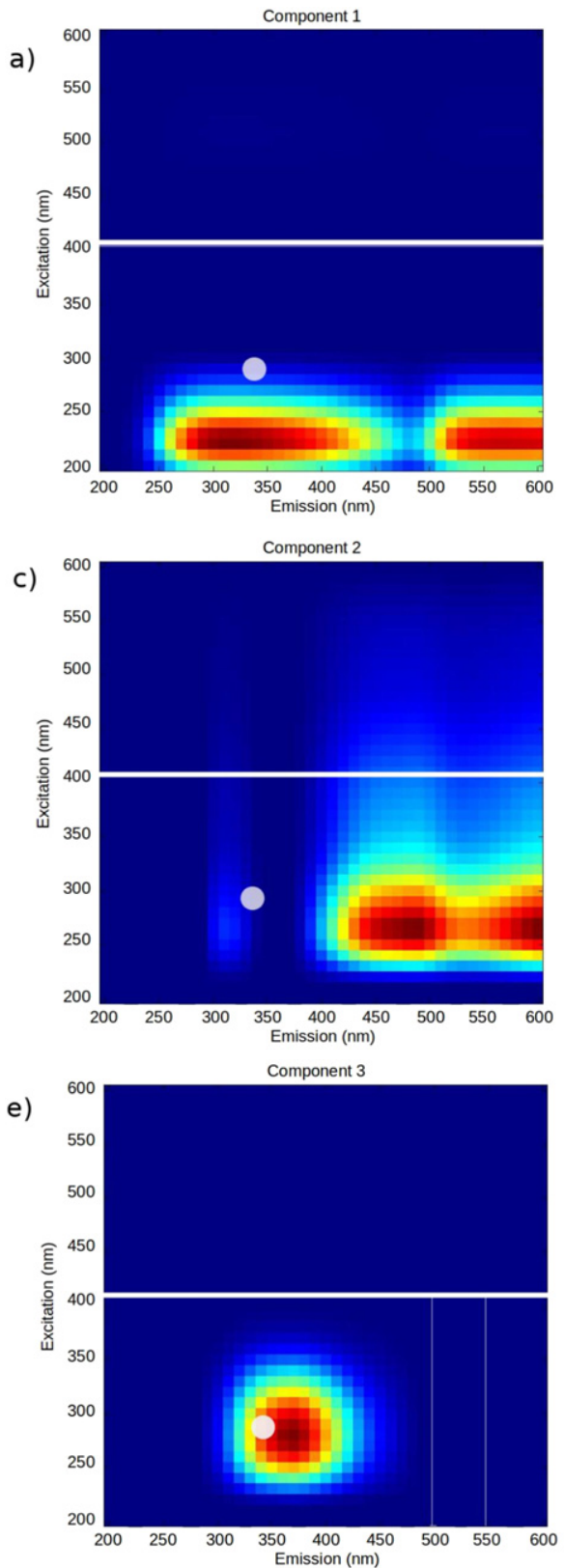

presents a two peaks pattern at $I^{C 1 T}{ }_{\text {max }}=240 / 340 \mathrm{~nm}$ and $I^{\prime C 1 T}{ }_{\text {max }}=$ $240 / 550 \mathrm{~nm}$. This component could be related to TRP response or boric acid noise. The second component presents also a double peak at $I^{C 2 T}{ }_{\text {max }}=260 / 450 \mathrm{~nm}$ and $I^{\prime C 2 T}{ }_{\text {max }}=260 / 600 \mathrm{~nm}$. One could make the hypothesis that this component $\left(\mathrm{C}_{2}\right)$ is related to the humic substance response, particularly concerning the first peak. The third component presents a single maximum $I^{\mathrm{C} 3 T}{ }_{\max }=290 / 360$ and could also be related to a proteinic signal of TRP, as its maximum fits well with the TRP fluorescence maximum in solution.

The same data treatment applied on $\{\mathrm{QS}-\mathrm{BA}\} ;\{\mathrm{QS}-\mathrm{HA}\}$ systems gives the components presented in Fig. 2, and are called $\mathrm{C} 1_{\mathrm{B}}, \mathrm{C} 2_{\mathrm{B}}$ and $C 3_{B}$ respectively. The first component $C 1_{B}$ is similar to the $C 1_{T}$. This component could be linked to the boric acid matrix effect or response as it is common to $\{\mathrm{QS}-\mathrm{BA}\} ;\{\mathrm{Q} S-\mathrm{HA}\}$ and $\{\mathrm{TRP}-\mathrm{BA}-\mathrm{HA}\}$. The second component $\mathrm{C} 2_{\mathrm{B}}$ presents a double peak at $I^{\mathrm{C2B}}{ }_{\max }=$ $250 / 380 \mathrm{~nm}$ and $I^{I^{C 2 B}}{ }_{\max }=250 / 600 \mathrm{~nm}$. This second peak, $I^{I^{C 2 B}}{ }_{\max }$,

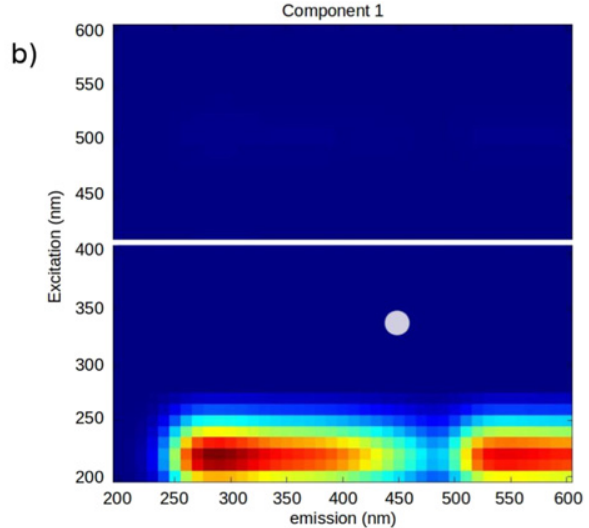

d)
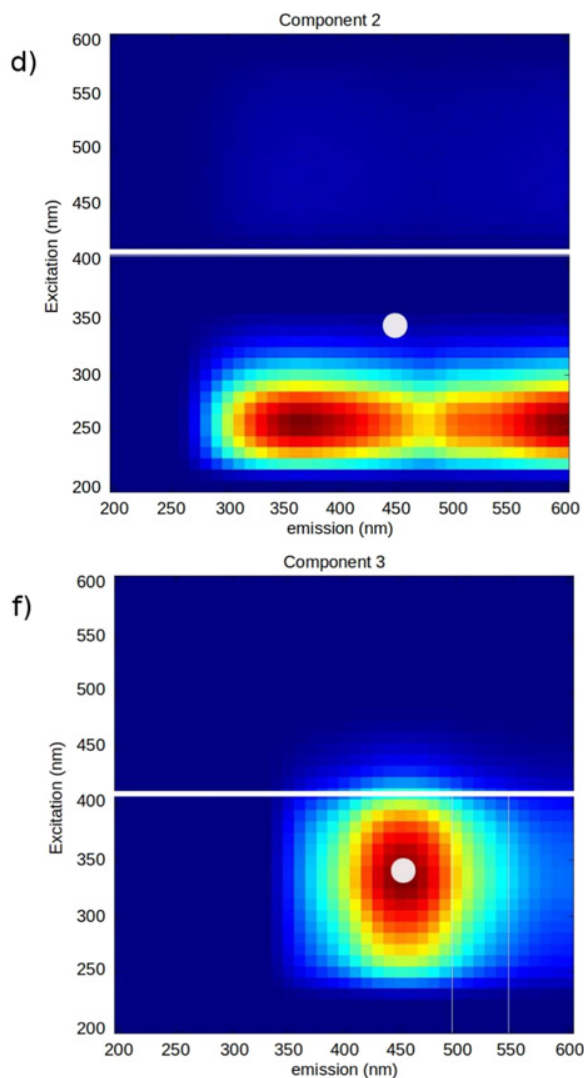

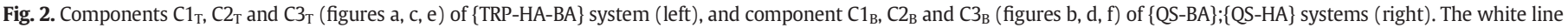

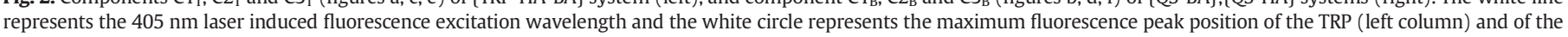
QS (right column) in solution. 
seems to be common to the $I^{\prime C 2 T}{ }_{\max }$. The third component is located at $I_{\text {max }}^{C 3 B}=340 / 450 \mathrm{~nm}$ which is different from the TRP experiment, and fits well with the fluorescence maximum of QS in solution.

The distribution of the component is represented in ternary diagrams Fig.3. The behavior of the CP/PARAFAC response versus the TRP content is not linear. Component C1T and C2T behave similarly, presenting several maximum intensity zones. Then mixture with $25 \%$ of $\mathrm{HA}$ presents 3 zones of maximum intensity respectively at 15, 60 and 80\% of TRP. Over $25 \%$ of HA, the signal decrease drastically and no contribution was observed for $100 \%$ of HA. At $0 \%$ of HA, two maximum zone are observed, one at $15 \%$ and another at $60 \%$ of TRP. A minimum of contribution is observed in the $15 \%$ of $\mathrm{HA}$ band. The $\mathrm{C}_{\mathrm{T}}$ shows contribution maximum for the $100 \%$ BA point, and a decreasing contribution when TRP and HA content increase. Hence, no linear behavior is observed for the TRP, HA or BA content as expected. Moreover, the response of component $\mathrm{C}_{\mathrm{T}}$ and $\mathrm{C} 2_{\mathrm{T}}$ is not specific (column 1, lines 1 and 2 , white circle, Fig. 2). If the $\mathrm{C}_{\mathrm{T}}$ is considered as linked to the TRP response, no linear behavior is observed, and a strong HA inner effect could be observed (Fig. 3c).

The Fig. (4) shows the results for CP/PARAFAC components contribution for the binary experiments $B$ (column 1 ) and the similar graph for experiment $\mathrm{T}$ (column 2), extracted for ternary diagrams. HA content of $0 \%, 0.1 \%, 0.5 \%$ and $0 \%, 2 \%, 10 \%$ were represented for QS and TRP respectively. For the QS system (Fig. 4a, c and e), there is an increase of contribution of component $\mathrm{C} 1_{\mathrm{B}}$ and $\mathrm{C} 2_{\mathrm{B}}$ from 0 to $20 \%$ of $\mathrm{QS}$, and an enhancement of the $0.1 \%$ of HA. For a content of $0.5 \%$ of $\mathrm{HA}$, there is a total extinction of the signal. For the $\mathrm{C}_{\mathrm{B}}$ component, the most similar to QS fluorescence, an increase is observed for $0 \%$ to $10 \%$, with a valley at $20 \%$ and again an increase that slow down to a plateau. For this component, the enhancing effect of HA is not observed, but the inner filter effect is still present. The contribution does not behave linearly and is highly perturbed. In the case of the TRP extracted data (Fig. 4b, $d$ and f), the 3 components, $\mathrm{C} 1_{\mathrm{T}}, \mathrm{C} 2_{\mathrm{T}}$ and $\mathrm{C} 3_{\mathrm{T}}$, behave similarly, with an increase trend from $0 \%$ to $5 \%$ of TRP, reaching a plateau. No enhancement of fluorescence is observed for the $2 \%$ HA content, and no total extinction of the signal is observed at $10 \%$ of HA content.

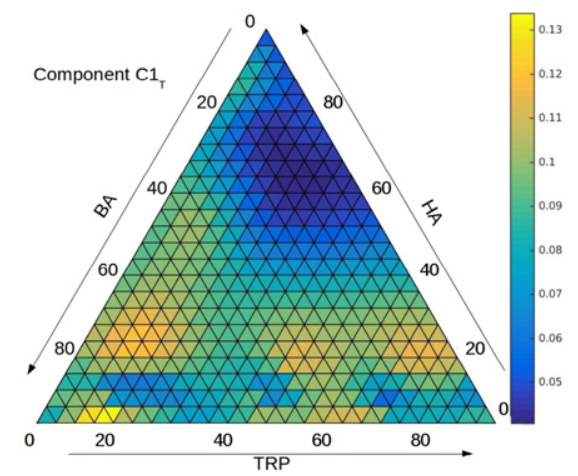

The FFFS response does not behave as a linear model and a strong extinction effect occurs when HA is present. In addition to quenching effect, energy transfer could occur in the solid. This could be an hypothesis for the rise of the $C 1_{B}$ and $C 2_{B}$ when HA content slightly increases, while no fluorescence is observed for pure HA. Finally none of the components behave as normal, and HA content highly perturb the expected linear trend.

This observed behavior of FFFS response is in accordance with results from [20] who found no 3D fluorescence response for organic matter, but in contradiction with another work which deals with direct 3D Frontal Fluorescence [1]. These differences should be due to sample preparation (raw, grind, powder, pellet...) or measurement processes (angle, apparatus, optical parameter, inner filter effect...) and point out the critical problem of standardization of this recent application of 3D FFFS. Theses authors do not specify well details of their experiments, but it seems that for some of them they use quartz cell filled with sample powder instead of pellets.

\subsection{Laser Induced Fluorescence Results}

The normalized to under curve area spectra of the fluorescence induced by laser are presented on top line (a) of the Fig. (5) for samples containing only TRP, QS or HA respectively. The second line (b) represents the $I F^{L I F}{ }_{405}$ for different TRP and QS contents in presence of different amount of HA, and in function of the HA content. The $I F^{L I F}{ }_{405}$ response seems to behave linearly to the TRP and QS contents considering integration of the curve response. The $I F_{405}^{L I F}$ is higher for the QS than for the TRP. There is a quenching effect for both TRP and QS when HA is added (Fig.5). However, concerning the HA, there is no response, even more, there is an exponential decreasing trend with the increasing content of HA (Fig. 5f). One can observe that for both TRP and QS there is no self inner effect or saturation of the LIF response. The normalized emission spectra for all the mixtures shows that there is two principal contribution to the LIF signal, one around $\lambda_{\mathrm{em}}=480 \mathrm{~nm}$ of emission that appears whatever the molecules, and one around $\lambda_{\mathrm{em}}=525 \mathrm{~nm}$ that appears as a shoulder when TRP is present.

b)

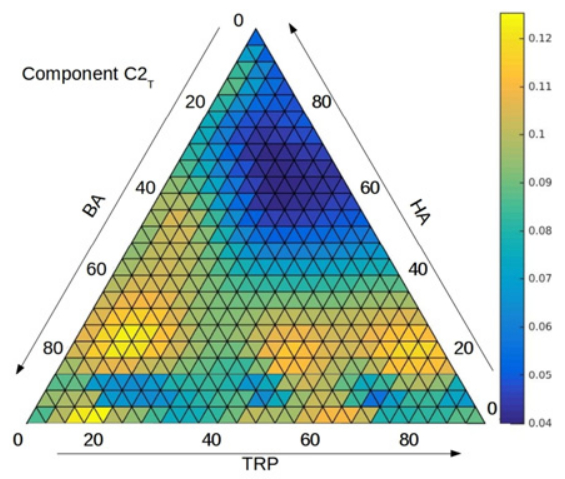

c)

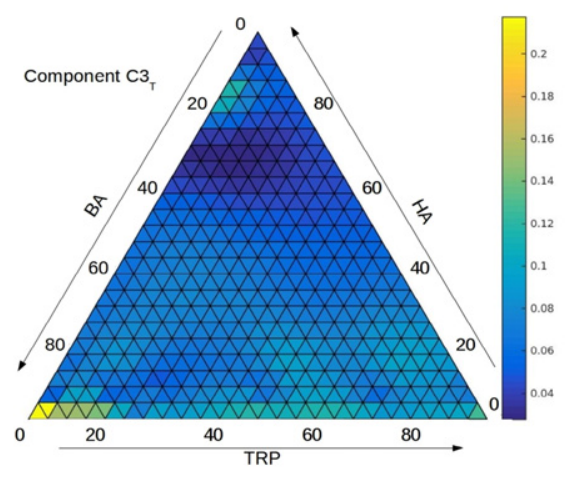

Fig. 3. Ternary contribution of $\mathrm{CP} / \mathrm{PARAFAC}$ components $\mathrm{C} 1_{\mathrm{T}}, \mathrm{C} 2_{\mathrm{T}}$ and $\mathrm{C} 3$ (a, b, c respectively) for \{TRP-HA-BA\} ternary system. Color scale represents the component contribution. 
a)

C1 contrib. vs $\{\mathrm{QS}\}$

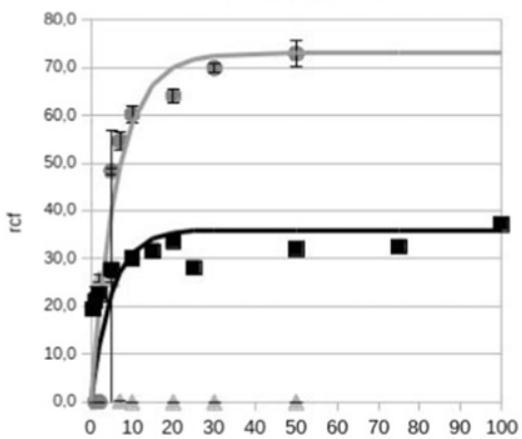

(QS) \%

c)

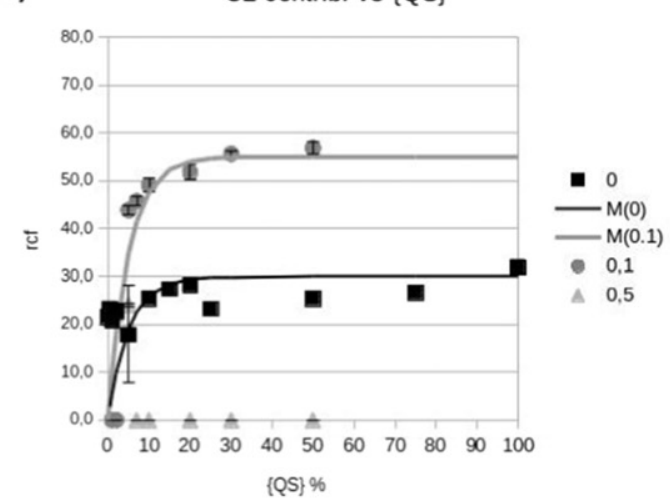

e)
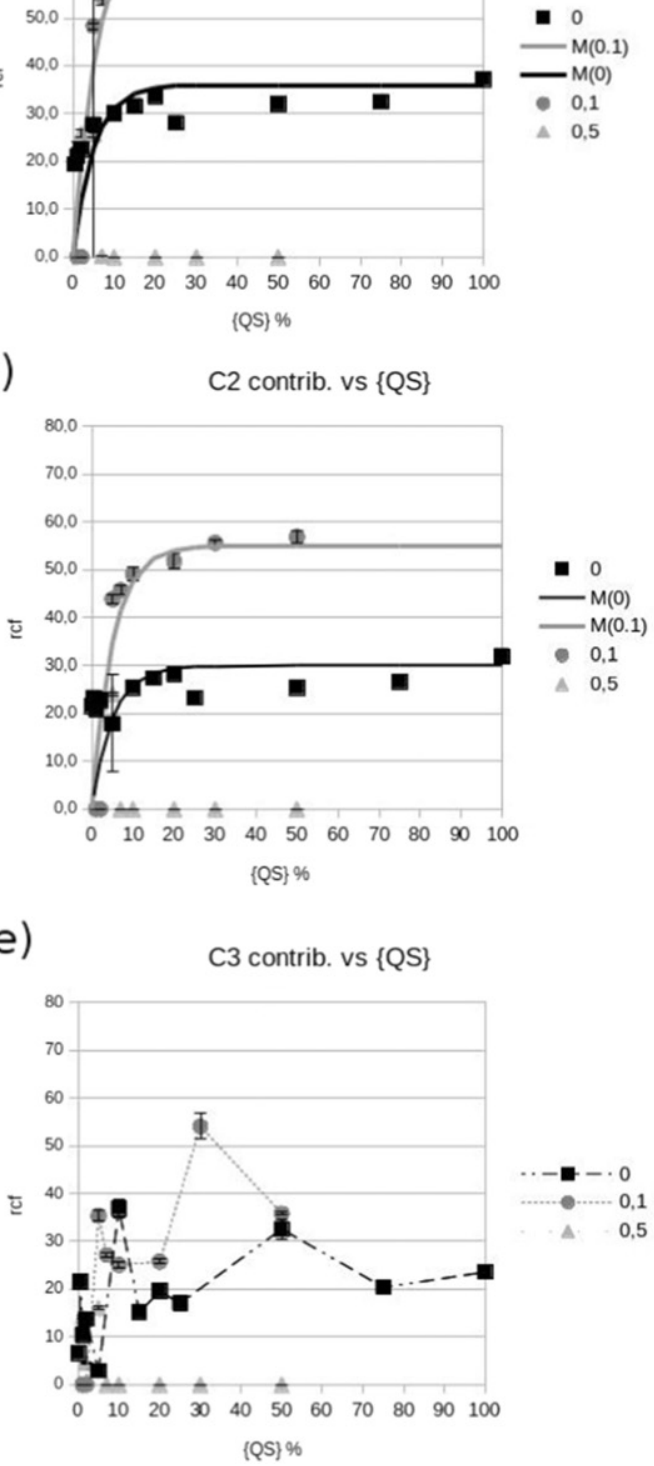

C2 contrib. vs $\{\mathrm{QS}\}$ b) C1 contrib. vs $\{$ TRP $\}$

d)

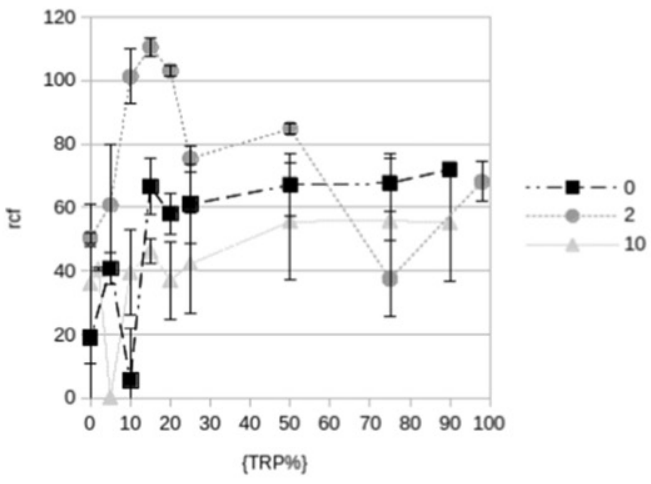

C2 contrib. vs $\{$ TRP $\}$

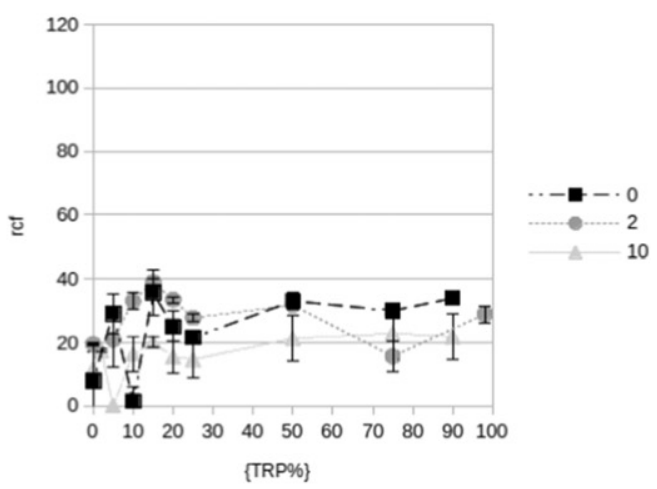

f)

C3 contrib. vs $\{$ TRP $\}$

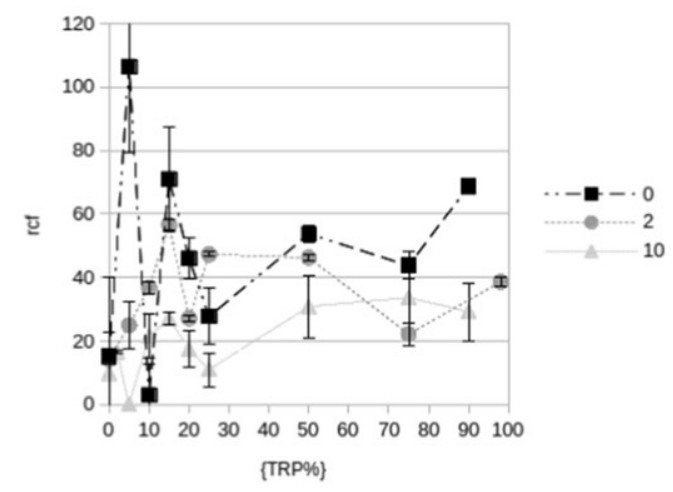

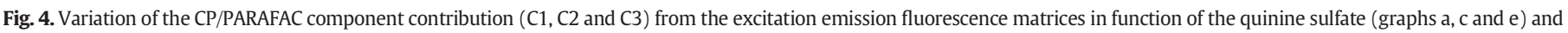
the tryptophan (graphs b, d and f) in presence of different amounts of humic acid.

Considering the $\mathrm{CP} /$ PARAFAC components $\mathrm{C} 3_{\mathrm{T}}$ and $\mathrm{C} 3_{\mathrm{B}}$, those which are close to the normal fluorescence properties of respectively TRP and QS (Fig. 2, line 3, white circle), no wavelength interaction seems possible for an excitation at $\lambda_{\mathrm{ex}}=405 \mathrm{~nm}$ and an emission at $\lambda_{\mathrm{em}}=480 \mathrm{~nm}$ for TRP. For SQ, $\lambda_{\text {ex }}=405$ could slightly interact with the $C 3_{B}$, but the $\lambda_{\mathrm{em}}=525 \mathrm{~nm}$ from LIF does not correspond. For the $\lambda_{\mathrm{em}}=480 \mathrm{~nm}$, this peak seems to be present whatever the sample. In the case of HA, it seems that the emission peak at $\lambda_{\mathrm{em}}=480 \mathrm{~nm}$ decreases with the increase of the HA content (Fig. 5). In case of LIF measurement, a good correlation exists between content of QS $\left(r^{2}>0,96\right)$ or TRP $\left(r^{2}>0,95\right)$ and $I F^{L I F}{ }_{405}$, but no specificity of the emission spectra is observed. This could prevent the characterization of QS and TRP if they are mixed together in a solid sample. Moreover, it should be taking in account the possible inner filter effect that occurs in presence of matrix different than BA. This was already pointed out in the case of acetyl salicylic acid determination in medical where the matrices lead to a shift of the calibration curve [3].

\subsection{Response Model}

The FFFS response in LIF or EEM mode lead to a saturation behavior that can be modeled by a very simple model. If we consider the surface of the sample, the photon flux reaching the surface is constant during time and proportional to the I0 intensity. Assuming that the two techniques analyze only the surface emission spectra, the probability for a photon to reach a molecule depends on the number of molecules present at the exposed surface, which can be linked to the molecule content on the solid called C and expressed in \% of mass. Using the Beer Lambert law, one can assume that the number of absorbed photon is described by the following formula:

$\mathrm{I}_{\mathrm{a}}=\mathrm{I}_{0} \cdot\left(1-e^{-\varepsilon /\left(\lambda_{\mathrm{ex}}\right) \cdot \mathrm{ll} \mathrm{c}}\right)$ 


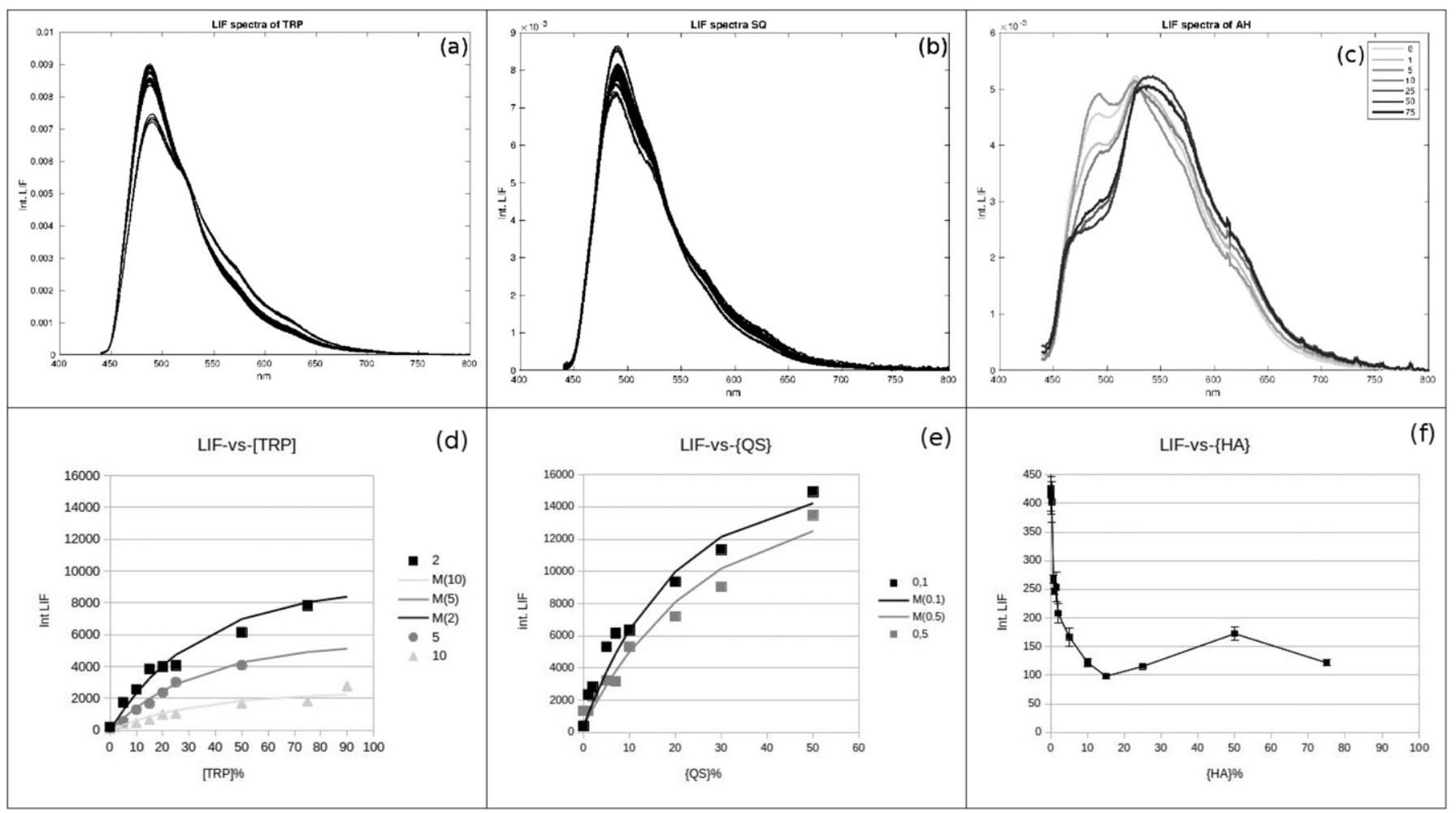

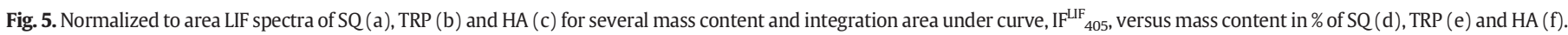
Solid lines represent the empirical model for each case except the HA curve where it is eyes guide.

With $\varepsilon^{\prime}\left(\lambda_{\mathrm{ex}}\right)$ the absorption coefficient related to molecule content in $\%$ of mass in $\mathrm{m}^{-1} \cdot \%^{-1}$ at $\lambda_{\text {ex }}$, l the path length in meter, and $\mathrm{C}$ the molecule content in \% of mass, $\mathrm{I}_{\mathrm{a}}$ the absorbed intensity and $\mathrm{I}_{0}$ the incident intensity.

The observed fluorescence is proportional to the quantum yield of fluorescence and, considering that there is no inner filter effect as the fluorescence emission is directed to the exterior, the fluorescence response is given by:

If $=\Phi \cdot \mathrm{K} \cdot \gamma\left(\lambda_{\mathrm{em}}\right) \cdot \mathrm{I}_{0} \cdot\left(1-e^{-\varepsilon /\left(\lambda_{\mathrm{ex}}\right) \mathrm{I} \cdot \mathrm{C}}\right)$

With $\Phi$, is the fluorescence quantum yield of the molecule, $\gamma\left(\lambda_{\mathrm{em}}\right)$ the probability of emission at $\lambda_{\mathrm{em}}$ and $\mathrm{K}$ an experimental factor linked to the efficiency to measure the emitted signal by the detector.

This simple model allows reproducing the behavior of the LIF response of QS $\left(r^{2}>0,96\right)$ and TRP $\left(r^{2}>0,95\right)$ (SI, (Fig. 7)), and in a some extend of the $\mathrm{C}_{\mathrm{B}}\left(\mathrm{r}^{2}>0,90\right)$ and $\mathrm{C} 2_{\mathrm{B}}\left(\mathrm{r}^{2}>0,77\right)$ contribution. However, one can observe that even if the path length could be estimated very small, the $\varepsilon^{\prime}\left(\lambda_{\mathrm{ex}}\right)$.l.C could not be considered as negligible.

\section{Conclusion}

In this work it was tested two FFFS techniques to measure the content of two simple molecules in presence of standard humic substances. The results show that in boric acid matrix, the detection of TRP and QS is possible using laser induced fluorescence, but without emission spectra specificity as the observed induced fluorescence emission positions do not fit with the TRP or QS fluorescence properties in solution. This could be due to the wavelength of excitation used and the energy involved in the process. Nevertheless, it has been shown that in other matrix like bovine albumin (BSA) or in starch, semi-quantitative measurements ever could be done. The simple model of evolution of LIF response and some CP/PARAFAC component contribution explains partly the behavior of the response by a surface saturation phenomena, but depending on molecules (TRP, QS, HA) and instrumentation (LIF, EEMs). Interestingly, the role of the humic substances has been restricted to inner filter effect of TRP or QS fluorescence response. No specific fluorescence signal nor by LIF-FFFS or EEM-FFFS was observed. Even more, when only humic acid is present in boric acid, the emission response decrease with the HA content for LIF, meaning that the scattering process in boric acid is quenched or absorbed by the HA. More investigation have to be done to understand complex interaction of organic molecules and minerals when they are excited by incident wavelength, but one important point is that for soils samples, the direct measurement of solid organic matter still be a real challenge as it behaves more as a quencher than a fluorescent molecule. Moreover, it is recommended is future works on FFFS to well inform the sample preparation and conditions of measurement (geometry, humidity, solid state, ...) as it could lead to different results as found in previous researches.

\section{Acknowledgements}

This work was supported by the Professor Visitante Especial CNPq/ Ciença sem Fronteira on Fluorescence Response on Heterogeneous Surfaces [processo no. 313576/2013-0] and the FAPESP [grant no. 2012/ 24349-0]. Special acknowledgements to Zhang Fu who helped a lot in the experimental part of this work.

\section{Appendix A. Supplementary data}

Supplementary data to this article can be found online at http://dx. doi.org/10.1016/j.saa.2017.01.017.

\section{References}

[1] R. Albrecht, E. Verrecchia, H.R. Pfeifer, The use of solid-phase fluorescence spectroscopy in the characterisation of organic matter transformations, Talanta 134 (2015) 453-459. 
[2] I. Allais, R.B. Edoura-Gaena, É. Dufour, Characterisation of lady finger batters and biscuits by fluorescence spectroscopy-relation with density, color and texture, J. Food Eng. 77 (4) (2006) 896-909.

[3] J.C.L. Alves, R.J. Poppi, Pharmaceutical analysis in solids using front face fluorescence spectroscopy and multivariate calibration with matrix correction by piecewise direct standardization, Spectrochim. Acta A Mol. Biomol. Spectrosc. 103 (2013) 311-318.

[4] F. Ammari, R. Bendoula, D.J.-R. Bouveresse, D.N. Rutledge, J.M. Roger, 3D front face solid-phase fluorescence spectroscopy combined with independent components analysis to characterize organic matter in model soils, Talanta 125 (2014) 146-152.

[5] F. Ammari, C.B.Y. Cordella, N. Boughanmi, D.N. Rutledge, Independent components analysis applied to 3D-front-face fluorescence spectra of edible oils to study the antioxidant effect of Nigella sativa L. extract on the thermal stability of heated oils, Chemom. Intell. Lab. Syst. 113 (2012) 32-42.

[6] R. Bro, H.A.L. Kiers, A new efficient method for determining the number of components in PARAFAC models, J. Chemom. 17 (5) (2003) 274-286.

[7] R. Bro, PARAFAC. Tutorial and applications, Chemom. Intell. Lab. Syst. 38 (2) (1997) 149-171.

[8] R.F. Chen, Y. Jiang, M. Zhao, Solid-phase fluorescence determination of chlorins in marine sediments, Org. Geochem. 31 (12) (2000) 1755-1763.

[9] R.F. Chen, Fluorescence quantum yields of tryptophan and tyrosine, Anal. Lett. 1 (1) (1967) 35-42.

[10] P.G. Coble, Characterization of marine and terrestrial DOM in seawater using excitation-emission matrix spectroscopy, Mar. Chem. 51 (4) (1996) 325-346.

[11] R.N. Conmy, P.G. Coble, C.E. Del Castillo, Calibration and performance of a new in situ multi-channel fluorometer for measurement of colored dissolved organic matter in the ocean, Cont. Shelf Res. 24 (3) (2004) 431-442.

[12] C. Goletz, M. Wagner, A. Grübel, W. Schmidt, N. Korf, P. Werner, Standardization of fluorescence excitation-emission-matrices in aquatic milieu, Talanta 85 (1) (2011) 650-656.

[13] J.F. Holland, R.E. Teets, P.M. Kelly, A. Timnick, Correction of right-angle fluorescence measurements for the absorption of excitation radiation, Anal. Chem. 49 (6) (1977) 706-710.

[14] A.B. Hougaard, A.J. Lawaetz, R.H. Ipsen, Front face fluorescence spectroscopy and multi-way data analysis for characterization of milk pasteurized using instant infusion, LWT Food Sci. Technol. 53 (1) (2013) 331-337.

[15] R. Karoui, E. Dufour, J. De Baerdemaeker, Monitoring the molecular changes by front face fluorescence spectroscopy throughout ripening of a semi-hard cheese, Food Chem. 104 (1) (2007) 409-420.
[16] L. Lakhal, V. Acha, T. Aussenac, PARAFAC analysis of front-face fluorescence data: Absorption and scattering effects assessed by means of Monte Carlo simulations, Chemom. Intell. Lab. Syst. 116 (null) (2012) 112-122.

[17] X. Luciani, S. Mounier, R. Redon, A. Bois, A simple correction method of inner filter effects affecting FEEM and its application to the PARAFAC decomposition, Chemom. Intell. Lab. Syst. 96 (2) (2009) 227-238.

[18] W.H. Melhuish, Quantum Efficiencies of Fluorescence of Organic Substances: Effect of Solvent and Concentration of the Fluorescent Solute, J. Phys. Chem. 65 (2) (1961) 229-235

[19] S.É. Mounier, H. Zhao, C. Garnier, R. Redon, Copper complexing properties of dissolved organic matter: PARAFAC treatment of fluorescence quenching, Biogeochemistry 106 (1) (2011) 107-116.

[20] M. Muller, D.M.B.P. Milori, S. Déléris, J.P. Steyer, Y. Dudal, Solid-phase fluorescence spectroscopy to characterize organic wastes, Waste Manag. 31 (9-10) (2011) 1916-1923.

[21] T. Ohno, Fluorescence inner-filtering correction for determining the humification index of dissolved organic matter, Environ. Sci. Technol. 36 (4) (2002) 742-746.

[22] D.M. Reynolds, Rapid and direct determination of tryptophan in water using synchronous fluorescence spectroscopy, Water Res. 37 (13) (2003) 3055-3060.

[23] C.H. Santos, G. Nicolodelli, R.A. Romano, A.M. Tadini, P.R. Villas-Boas, C.R. Montes, S. Mounier, D.M.B.P. Milori, Structure of humic substances from some regions of the Amazon assessed coupling 3D fluorescence spectroscopy and CP/PARAFAC, J. Braz. Chem. Soc. 26 (6) (2015) 1136-1142.

[24] J.T. Vivian, P.R. Callis, Mechanisms of tryptophan fluorescence shifts in proteins, Biophys. J. 80 (5) (May 2001) 2093-2109.

[25] L.T. Shirshova, A.L. Kholodov, B.N. Zolotareva, L.A. Fominykh, A.M. Yermolayev, Fluorescence spectroscopy studies of humic substance fractions isolated from permanently frozen sediments of Yakutian coastal lowlands, Geoderma 149 (1-2) (Feb. 2009) 116-123.

[26] C.A. Stedmon, S. Markager, Resolving the variability of dissolved organic matter fluorescence in a temperate estuary and its catchment using PARAFAC analysis, Limnol. Oceanogr. 50 (2) (2005) 686-697.

[27] G.R. Zepp, W.M. Sheldon, M.A. Moran, Dissolved organic fluorophores in southeastern US coastal waters: Correction method for eliminating Rayleigh and Raman scattering peaks in excitation-emission matrices, Mar. Chem. 89 (1-4) (2004) 15-36. 